\title{
Physique des \\ rayonnements et \\ sciences du vivant
}

Le CEA et

la recherche biomédicale,

un aperçu historique

Jean-François Picard

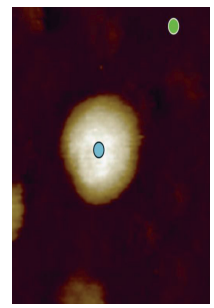

CNRS, 7 rue des Larris,

78460 Chevreuse, France.

jfmpicard@gmail.com

> Le rôle de la physique nucléaire dans l'essor des sciences de la vie au cours du vingtième siècle est un fait bien documenté en histoire des sciences (voir par exemple $[1,2])$. En France, inscrit à la suite du prix Nobel 1935 décerné aux époux Joliot pour la découverte de la radioactivité artificielle et de ses applications en biologie, le Commissariat à l'énergie atomique (CEA) est installé en 1945 en vue de développer les usages industriels et militaires de l'énergie nucléaire et, accessoirement, de développer la production et l'usage des radioéléments issus de ses installations. Au début des années 1950, son département de biologie est chargé de fournir aux laboratoires qui en feront la demande, comme ceux de l'Institut Pasteur ou du CNRS, les radiomarqueurs produits dans ses accélérateurs de particules ainsi que l'instrumentation nécessaire à leur usage. La recherche médicale y est prise en compte, au début des années 1960, lorsque le service hospitalier Frédéric Joliot (SHFJ) s'installe à Orsay, à deux pas du CEASaclay, afin de développer les nouvelles techniques de l'imagerie médicale. Cependant, à la fin du vingtième siècle, bien qu'il soit devenu un partenaire incontournable de la recherche en sciences de la vie, les destinées du CEA semblent remises en cause par la fin des grands programmes d'équipements lancés lors de sa création, de l'industrie électronucléaire et de la mise

Vignette (Photo (c) Cendrine Faivre-Moskalenko) au point de l'arme atomique. Ce que l'on voudrait évoquer ici est la manière dont la réorganisation de son secteur dédié aux sciences du vivant a non seulement préservé le Commissariat à l'énergie atomique et aux énergies alternatives, mais aussi comment elle servit de modèle pour réorganiser l'ensemble de la recherche publique effectuée en France dans ce domaine.

\section{Les prémisses}

La découverte de la radioactivité par Henri Becquerel, Pierre et Marie Curie n'a pas attendu la sanction des Nobel de 1903 et 1911 pour donner lieu aux premières applications médicales. À l'aube du vingtième siècle, à I'hôpital Saint-Louis à Paris, Henri Alexandre Danlos semble avoir été le premier dermatologue à utiliser du radium pour soigner un carcinome. En 1909, Madame Curie obtient que I'Institut Pasteur et l'université de Paris installent sur la montagne SainteGeneviève un Institut du radium destiné à combattre le «fléau des temps modernes», le cancer [3]. En 1924, Frédéric Joliot y est recruté et il épouse Irène, la fille et assistante de la patronne. Les travaux des deux chercheurs aboutissent à la découverte de la radioactivité artificielle, récompensée par un nouveau Nobel en 1935. Dans la conférence qu'il fit à Stockholm, Frédéric Joliot évoque les applications de cette découverte: « [...] comme la méthode des marqueurs, permise par la disposition des radioéléments synthétiques introduits 
dans des organismes vivants pour les étudier ou pour les soigner », mais il n'omet pas d'autres éventualités, « [...] comme de pouvoir domestiquer la fantastique énergie de la matière, voire d'imaginer des effets beaucoup plus destructeurs ${ }^{1}$. Devenu une étoile de la communauté scientifique, Joliot veut s'insérer dans la compétition internationale à l'aube de la big science. Élu au Collège de France en 1936, il quitte l'ambiance un peu artisanale de l'Institut Curie et, grâce au soutien de la fondation Rockefeller et d'un CNRS en cours d'organisation, il obtient la construction d'un cyclotron et l'installation d'un laboratoire de synthèse atomique (le LSA), à Ivry en banlieue parisienne. En 1939, à la suite de la découverte du phénomène de fission par 0 . Hahn, F. Strassmann et L. Meitner, Joliot et ses assistants, Lew Kowarski et Hans v. Halban expérimentent le processus d'une réaction en chaîne, ce qui aboutit au dépôt de plusieurs brevets CNRS à la veille de la Seconde Guerre mondiale dont I'un pour un «dispositif de production d'énergie » et l'autre pour des «perfectionnements aux charges explosives » [4].

Au laboratoire de synthèse atomique, en 1937, Frédéric Joliot a reçu la visite du chimiste Georg v. Hevesy (Prix Nobel 1943), le pionnier de l'utilisation des radio-isotopes en biologie. La fabrication et l'utilisation de radioéléments artificiels se développent en effet rapidement et près de 125 mémoires scientifiques s'y rapportant sont publiés dans le monde entre 1937 et 1940 [5]. Pendant la guerre, lui et ses collègues biologistes du Collège de France, Robert Courrier et Antoine Lacassagne, étudient la fixation du radio-iodé dans la thyroïde. En 1943, dans une conférence donnée alors qu'il vient d'être élu à l'Académie des Sciences, Joliot évoque ces travaux : «C'est probablement en biologie que les radioéléments rendront les plus grands services, notamment dans l'étude du métabolisme thyroi-dien... Par exemple, la méthode autoradiographique permet de préciser la localisation précise des substances dans l'organisme vivant en réalisant l'image d'un tissu » [6]. Pour l'heure, ces applications semblent moins pertinentes en médecine qu'en biologie, note le Docteur Antoine Lacassagne que son intérêt pour la recherche a éloigné de la clinique. Dans les années 1930, à l'Institut du radium, Lacassagne avait abandonné son activité hospitalière, ulcéré par l'irresponsabilité de ses confrères capables de faire absorber des substances radioactives à leurs patients hors du simple bon sens thérapeutique [7]. À la Libération, alors qu'il vient de prendre la direction de l'Institut du radium, il participe aux travaux d'une commission de réforme de la recherche médicale mise en place au CNRS, I'organisme étant alors dirigé par Joliot. Devant cette commission, il préconise la démédicalisation de la recherche médicale au profit des seuls biologistes, une mesure qu'il justifie, en évoquant: «Le désintérêt des facultés de médecine pour les plus récentes avancées dans les sciences de la vie et par l'archaïsme d'hôpitaux qui ignorent l'activité de laboratoire »?'.

\section{Le Commissariat à l'énergie atomique et la biologie}

Alors que la bombe d'Hiroshima marque la fin de la Seconde Guerre mondiale, le Général de Gaulle décide l'installation d'un Commissariat

${ }^{1}$ http://www.nobelprize.org/nobel_prizes/chemistry/laureates/1935/joliot-fred-lecture.html

${ }^{2}$ Lacassagne A, Comment développer en France la recherche scientifique dans le domaine de la médecine expérimentale? Note datée d'octobre 1944 (archives CNRS, AN-80-284). à l'énergie atomique (CEA) dont il confie la direction à Frédéric Joliot et à l'ancien ministre de l'armement Raoul Dautry. L'ordonnance du 18 octobre 1945 stipule les missions du nouvel organisme: «Poursuivre les recherches scientifiques et techniques en vue de l'utilisation de l'énergie atomique dans les divers domaines de la science, de l'industrie et de la défense nationale $»^{3}$. Sollicité par un engagement politique de plus en plus affiché au sein du Parti communiste, Joliot laisse une large initiative aux équipes chargées d'organiser le nouvel organisme, notamment à Lew Kowarski qui entreprend de construire une première pile atomique, au fort de Chatillon en banlieue parisienne. La pile ZOE (zéro énergie, oxyde d'uranium, eau lourde) diverge ${ }^{4}$ le 15 décembre 1948, un événement médiatisé dans la presse de l'époque comme un symbole du redressement national qui s'accompagne de la mise en place d'un service de médecine du travail pour assurer la protection du personnel chargé de son fonctionnement ${ }^{5}$.

Simultanément, le comité scientifique du Commissariat confie à Robert Courrier, Antoine Lacassagne et Louis Bugnard, le soin d'installer le service ad hoc au CEA afin d'y développer des recherches en radiobiologie. Louis Bugnard est un médecin polytechnicien, professeur de biophysique à la faculté de Toulouse, qui a participé au comité scientifique du CNRS avant-guerre aux côtés des époux Joliot. En 1946, il sollicite son recrutement par le Commissariat mais se voit suggérer d'accepter plutôt la direction de l'Institut national d'hygiène (INH) devenue vacante $^{6}$. En relation avec la Fondation Rockefeller, Bugnard inaugure, à l'INH, un système de bourses qui permet d'envoyer outre-Atlantique de jeunes internes intéressés par les développements d'une «biomedicine » alors en plein essor. Parmi eux, Maurice Tubiana se rend chez David Rittenberg à l'université Columbia puis participe, à son retour, à l'installation d'un bêtatron AllisChalmers $^{7}$ au centre anticancéreux de Villejuif ${ }^{8}$. Quant à Jean Coursaget, il est envoyé à la Mayo Clinic (Min-

${ }^{3}$ Le texte de l'Ordonnance est publié dans le premier rapport d'activité du CEA, 1945-1952 (archives CEA-FAR).

${ }^{4}$ Quand la réaction en chaîne est amorcée, on dit que la pile diverge, à cause de la forme que prend la courbe d'émission de neutrons.

${ }^{5}$ L. Kowarski visite le centre atomique de Harwell pour s'informer des mesures de sécurité prises par les Anglais autour de leurs installations. II suscite la création d'un service de médecine du travail, ainsi que la mise au point et la diffusion de dosimètres individuels. Recruté par le CEA, le Dr. Vidal se charge de vérifier l'état de santé du personnel appelé à travailler sur les installations de Châtillon, de l'usine du Bouchet et dans la mine d'uranium de La Crouzille. CEA, comité scientifique, 20 janvier 1948 (archives CEA-FAR).

${ }^{6}$ CEA, comité scientifique, 2 mai 1946 (archives CEA-FAR).

${ }^{7}$ Situé à l'Institut G. Roussy, cet accélérateur de particules est à usage médical ; il a été livré par la société Allis-Chalmers.

${ }^{8}$ Archives de l'Inserm, A. N. versement 760215. 
neapolis) où fonctionne le premier cyclotron ${ }^{9}$ à usage médical installé dans le monde. À son retour en France, Bugnard obtient de la Fondation Rockefeller la fourniture d'un spectroscope de masse, et du carbone 13, installé à l'hôpital Necker. En effet, le patron de l'INH se dit soucieux de son autonomie vis-à-vis de ses collègues Courrier et Lacassagne auxquels il reproche de «récupérer de manière exclusive au profit de l'Institut du radium, les isotopes produits par le cyclotron de Joliot au Collège de France » [8-10]. Ainsi en 1952, Jean Coursaget se voit proposer la direction du service de biologie au CEA, ce qui le conduit à préciser les recherches qu'il entend y mener : « $\varepsilon$ n matière de biologie, il y avait deux grandes possibilités, utiliser les radio-isotopes comme marqueurs moléculaires et considérer l'action des radiations sur le vivant, c'est-àdire faire de la radiobiologie. Une autre possibilité concernait la radioprotection mais cela ne nous intéressait pas car relevant d'une recherche appliquée pas très intéressante $»^{10}$.

Pour répondre à cette préoccupation jugée mineure par les chercheurs, le CEA installe en 1951 un Service de protection contre les radiations (SPR) dont la direction est confiée au Docteur Henri Jammet. Rattaché au centre de recherches du service de santé des armées, au fort de Chatillon, le SPR est chargé d'étudier les doses maximales de radiations admissibles par l'homme et de mettre au point les techniques et le traitement des irradiations accidentelles. Comme le Docteur Jammet a su nouer de bons contacts avec les cliniciens, notamment avec ses confrères cancérologues, à la suite de l'irradiation accidentelle de techniciens yougoslaves qui avait eu lieu à Vinca (Yougoslavie) en 1958, il participe avec Georges Mathé aux premières greffes de moelle osseuse destinées à combattre l'aplasie médullaire. Malgré des résultats parfois controversés, cette intervention représente une grande première en matière d'immuno-cancérothérapie [11]. Elle permettra à Mathé d'installer son Institut du cancer et d'immunogénétique à Villejuif (ICIG) et à Jammet de devenir une sommité au sein de la Commission internationale de protection radiologique (CIPR) [12].

\section{Une vocation pour le CEA, appareiller les sciences de la vie}

En 1954, dans un mémorandum adressé à Francis Perrin, le successeur de Joliot à la tête du CEA, Jean Coursaget justifie la place de la radiobiologie en soulignant que cet organisme « [...] est le seul à disposer de l'appareillage nécessaire à la fabrication des radioéléments indispensables à l'étude du métabolisme, mais dont le maniement requiert des précautions particulières, notamment pour la production de molécules marquées à vie brève, ce qui implique la proximité des sites de production et d'utilisation et (in fine) que cela ne devrait pas lui interdire de développer la recherche biologique dans les domaines où il est le mieux armé $»^{11}$. La mission princeps du département de biologie repose sur la fourniture de molécules marquées au radiocarbone (fructose, acide gluconique, glucose et ribose), voire sur le développement de nouveaux

\footnotetext{
${ }^{9}$ Le cyclotron est un accélérateur de particules qui utilise l'action combinée d'un champ électrique et d'un champ magnétique, afin d'accélérer et de confiner les particules dans un espace restreint.

${ }^{10}$ Témoignage de Jean Coursaget recueilli le 29 janvier 1997 (archives CEA-FAR).

${ }^{11}$ Biologie $(E A$, recherches à envisager, mémorandum de J. Coursaget à l'attention du Haut Commissaire F. Perrin, avril 1954 (archives CEA-Saclay).
}

radioéléments destinés aux laboratoires qui en feront la demande. Au centre de Saclay, André Menez développe l'utilisation de tritium, un isotope de l'hydrogène destiné à marquer de l'eau qui permet d'étudier la perméabilité des membranes biologiques. II s'agit aussi de mettre au point l'instrumentation nécessaire à l'acquisition des signaux émis par ces radioéléments, un domaine où le CEA ne se révèle pas moins performant. Que ce soit en radiocristallographie, en chromatographie, en microscopie électronique, en tomographie par émission de positons (TEP) ou en résonnance magnétique nucléaire (RMN), l'appareillage est conçu et réalisé dans ses laboratoires techniques de Grenoble ou de Saclay.

Ainsi dotés, les laboratoires du $C E A^{12}$ peuvent revendiquer quelques belles avancées scientifiques, ce qui permet à cet organisme de se poser en partenaire incontournable dans l'essor d'une nouvelle discipline, la biologie moléculaire. Grâce à une hormone tritiée, l'ocytocine, les physiologistes François Morel et Christian de Rouffignac analysent la filtration du sodium et la production d'urine dans le rein de lapins [13]. En biophysique, la mise au point d'un spectrographe éclair en spectrométrie Raman permet à deux ingénieurs agronomes, Eugène Roux et Pierre Guérin de Montgareuil, de décrypter, molécule par molécule, le processus de photosynthèse au centre de Cadarache en $1963^{13}$. Le responsable du service de biochimie, Pierre Fromageot, a constaté que l'utilisation de composés toxiques radiomarqués permet d'étudier la synthèse des protéines [14]; au début des années 1960, il recrute André Sentenac, un jeune biologiste frais émoulu de I'Institut national des sciences et techniques nucléaires (INSTN) pour s'intéresser à I'ARN polymérase de la bactérie Escherichia coli. Le service de biochimie du CEA est étroitement connecté avec l'Institut Pasteur, notamment avec les équipes de François Gros et de Jacques Monod qui travaillent sur la transcription de I'ADN en ARN et sa traduction en chaînes de polypeptides, les protéines. André Sentenac s'inscrit alors dans l'évolution qui voit la génétique délaisser les modèles bactériens, procaryotes, pour les cellules eucaryotes constitutives des organismes supérieurs. Ainsi, avec le centre de génétique moléculaire du CNRS à Gif-sur-yvette, il participe au programme européen de séquençage de la levure, réalisé grâce à la méthode autoradiographique qu'évoquait Joliot, un demi-siècle plus tôt [15]. Au début des années 2000, André Sentenac se voit confier la direction d'un Institut de génomique du CEA chargé

\footnotetext{
${ }^{12}$ En 1963, à l'occasion d'une réorganisation du CEA, le service de biologie devient un département rattaché à l'Institut de recherche fondamentale dirigé par le physicien Jules Horowitz.

${ }^{13}$ http://aprovenc. perso.neuf.fr/agronomie/pages/photosynthese.html
} 
de la tutelle du Centre national de génotypage (CNG) et du Génoscope (CNS) d'Évry ${ }^{14}$, au faîte d'une carrière couronnée par son élection à l'Académie des sciences.

\section{Le Service hospitalier Frédéric-Joliot (SHFJ) et l'essor de l'imagerie médicale}

Le succès limité des injections de radioéléments en cancérothérapie envisagée dans les années 1940 explique l'introduction plus tardive de la recherche médicale au CEA (la situation n'est pas spécifique à la France [16]). En juillet 1959, cela fait l'objet d'une note adressée à son comité de biologie: «[...] À la suite des travaux menés au Laboratoire de synthèse atomique deux décennies auparavant, y lit-on, un excès d'enthousiasme a pu laisser entrevoir un brillant avenir aux applications médicales de l'énergie nucléaire, notamment en exploitant l'action radiothérapique du rayonnement après concentration du radionucléide dans le tissu à détruire. Mais les progrès réalisés l'ont été au prix d'une complication instrumentale importante pour des résultats thérapeutiques limités et l'iode demeure le seul exemple à avoir pleinement répondu à cet espoir, grâce à sa fixation élective dans la glande thyroïde. S'il s'agit de développer la recherche médicale au CEA, conclut ce document, mieux vaudrait donc s'intéresser aux techniques diagnostiques qu'à celles de la radiothérapie $\gg^{15}$. Au début des années 1960 , le Service hospitalier Frédéric-Joliot (SHFJ) s'installe à l'hôpital d'Orsay sous la direction de Claude Kellershohn, spécialiste de médecine nucléaire à la faculté de Nancy. Deux options se présentent alors au SHFJ, soit faire de la radio-immunologie, c'est-à-dire le dosage hormonal qui vaudra à Rosalyn Yalow le Nobel de 1977, soit se consacrer à l'imagerie isotopique innovante, la voie finalement retenue par Claude Kellershohn, un pionnier de la tomographie par émission de positons (TEP). La TEP requiert la disposition de marqueurs isotopiques de périodes très courtes, ce qui amène le SHFJ à se doter d'un petit cyclotron fabriqué au CEA tandis que le développement des gamma caméras nécessaires à l'acquisition des signaux est confiée au Laboratoire d'électronique et de technologie de l'information de Grenoble (LETI). En utilisant de l'iode 132, cette instrumentation permet à Claude Kellershohn et Dominique Comar de mettre au point le diagnostic de l'hyperthyrö̈die congénitale [17]. En cardiologie, elle sert à filmer la cinétique de la circulation sanguine dans les oreillettes cardiaques, tandis que l'emploi d'or radioactif colloïdal comme marqueur, autorise l'examen du foie. En 1970, le SHFJ revendique la réalisation, en Europe, des premières images scintigraphiques de rein humain $^{16}$. La TEP est aussi à l'origine d'importants progrès en pharmacodynamique, notamment au Laboratoire d'étude du métabolisme des médicaments (LEMM) de Michel Istin à Saclay, puisqu'elle permet de suivre une molécule médicamenteuse jusqu'à son action sur un récep-

\footnotetext{
${ }^{14}$ Dirigé par Jean Weissenbach, le Génoscope - Centre national de séquençage a séquencé le chromosome 14 dans le cadre du Human Genome Project (2002).

${ }^{15}$ Les applications médicales des sciences et techniques nucléaires, note adressée au Haut commissaire Francis Perrin, datée du 27 juin 1959 (arch. CEA-Saclay).

${ }^{16}$ Le SHFJ acquiert rapidement une solide réputation en matière d'investigations cliniques. De 1960 à 1970, il a reçu près de 3000 patients dans ses 14 lits d'hospitalisation, examinés pour un tiers en cardiologie, un quart en hépatologie, $15 \%$ en thyroïdite et $9 \%$ en néphrologie. CEA, département de biologie. Rapport d'activité 1970 du SHFJ (archives A. Syrota).
}

teur. Plus tard, grâce à l'installation à l'université de Caen d'un cyclotron à usage médical (Cyceron), Bernard Mazoyer étudiera l'activité métabolique du système nerveux central, un champ d'activité appelé aux remarquables développements que l'on sait.

L'autre technique d'imagerie qui a été développée au $C \varepsilon A$, est la résonance magnétique nucléaire (RMN). La RMN était utilisée en biologie pour étudier la structure des protéines ${ }^{17}$. II revint à André Syrota, un praticien de I'hôpital du Kremlin-Bicêtre et professeur à l'université Paris Sud, d'inaugurer son usage médical au SHFJ dont il prendra la direction en $1985^{18}$. Contrairement à la TEP, la RMN est une méthode d'investigation non invasive fondée sur les propriétés de spin atomique. Elle ne requiert donc pas l'injection de radio-marqueurs, mais implique la disposition de champs magnétiques très puissants. Avec le soutien de Jules Horowitz, le patron de la recherche fondamentale au Commissariat, André Syrota obtient le façonnage d'un aimant supraconducteur de 2 teslas pour installer un appareil de RMN corps entier à Orsay, en 1980. Sa mise au point laborieuse évoquée par Gilles Bloch, un jeune médecin polytechnicien recruté par ses soins, n'en annonce pas moins les remarquables développements de cette instrumentation aussi bien en usage clinique qu'au sein des laboratoires ${ }^{19}$.

\section{La Direction des sciences du vivant, un modèle pour réorganiser la recherche}

Au tournant des années 1990, trois décennies de recherche ont fait du CEA un opérateur majeur dans les sciences de la vie ${ }^{20}$. Outre la disposition des moyens matériels, sans commune mesure avec ceux dont disposent le CNRS ou l'Inserm, ce succès s'explique aussi par les dispositions d'une chaîne de décision réduite où il a su marier les préoccupations technologiques aux avancées de la recherche fondamentale. «Au CEA, il n'y a pas de chercheurs (comme au CNRS ou à l'Inserm), seulement des ingénieurs. C'est-à-dire des gens qui peuvent passer d'une fonction de recherche à une fonction opérationnelle, sans trouver cela dégradant pour autant » dit André Syrota ${ }^{21}$. Ainsi, Jacques Grassi, le responsable du service de pharmacologie et d'immuno-analyse du CEA

\footnotetext{
${ }^{17}$ https://fr.wikipedia.org/wiki/Andr\%C3\%A9_Menez

18 Entretiens avec André Syrota en 2011, 2013-14, disponibles sur http://www. histrecmed.fr/index.php? option $=$ com_content\&view $=$ article\&id $=270$ : syrotaandre\&catid $=8$ : entretiens

${ }^{19}$ Entretiens de Gilles Bloch avec l'auteur, 12 mars 2015.

${ }^{20}$ La part du budget de recherche consacrée aux sciences de la vie a plus que doublé de 1970 à 1980, passant de 400 MF à un 1 milliard de francs (constants), ce qui représente $12 \%$ du budget consacré à l'ensemble de la recherche fondamentale menée au Commissariat, pour un demi millier d'agents (CEA - Rapport d'activité 1987, archives (EA-FAR).

${ }^{21}$ André Syrota (CEA - Rapport d'activité 1987, archives (EA-FAR).
} 
Saclay, qui n'hésite pas à se présenter comme un plombier de la science, ajoute que "l'une des caractéristiques du CEA est que l'on peut y faire une carrière complète sans publier tout en étant parfaitement reconnu si on est capable d'amener des produits jusqu'à une phase d'exploitation industrielle $\gg^{22}$. Cela a amené le CEA à créer de nombreuses filiales, par exemple un Office des rayonnements ionisants (ORIS) producteur d'anticorps monoclonaux marqués destinés à la recherche et à l'industrie pharmaceutique. En revanche, à l'exception notable du Centre d'études nucléaires de Grenoble (CEN-G), son mode d'intégration vertical, ne l'a guère incité à développer ses relations avec les autres établissements scientifiques et la question se pose du devenir d'un organisme dont les objectifs, fixés à la Libération, arrivent à leur terme : l'armement atomique ou l'électronucléaire, désormais passés en d'autres mains.

Le CEA entre alors dans une phase de réorganisation mouvementée dont les sciences de la vie vont tirer le meilleur parti. En 1991, l'organisme se dote d'une Direction des sciences du vivant (DSV) confiée à Michel Suscillon, le successeur du physicien Louis Néel au CEN-G. Dans les années 1970, Suscillon y avait introduit les sciences de la vie via une coopération internationale réunissant l'European Molecular Biology Organization (EMBO), le CNRS et l'université Joseph-Fourier, autour du réacteur à haut flux de l'Institut Laüe-Langevin dont les sources de neutrons permettaient d'étudier la structure et l'organisation spatiale des macromolécules biologiques $^{23}$. Cette coopération inter-organismes mise en œuvre au CहN-G, mais longtemps négligée par l'ex-département de biologie, inspire André Syrota lorsqu'il prend la tête de la Direction des sciences du vivant en 1993. L'objectif est de fédérer les laboratoires de biologie du CEA qui travaillent «par et pour le nucléaire », dit-il, au sein d'instituts largement ouverts à la coopération avec des établissements scientifiques français et étrangers, l'EMBL (European molecular biology laboratory), le CNRS et I'Inserm, I'Institut Pasteur et I'Institut Curie, I'INRA (Institut national de la recherche agronomique), I'INRIA (Institut national de recherche en informatique et en automatique), les laboratoires universitaires et ceux de l'industrie, etc. ${ }^{24}$ Tel est le cas de l'Institut de génomique à Évry évoqué plus haut ou de l'Institut de biologie structurale (IBS) à Grenoble, dont les lignes de lumière de l'European Synchrotron Radiation Facility (ESRF) permettent de pénétrer au cœur moléculaire de la vie ou de faire du «drug design » pour l'industrie pharmaceutique [18]. L'affaire des prions permet à la Direction des sciences du vivant de prouver sa réactivité par rapport aux autres organismes, tout en valorisant ses compétences technologiques. Suite à la crise de la vache folle avec la crainte de transmission de la maladie de Creutzfeldt-Jakob à l'homme, André Syrota réactive le service de neurologie du Service de santé des Armées de Dominique Dormont à Fontenayaux-Roses, tandis que Jacques Grassi met au point à l'Institut de biologie et de technologies de Saclay (IBITECS) un test de dépistage des prions pathogènes. Diffusé par Bio-Rad, l'efficacité de ce test permet au CEA d'engranger $70 \mathrm{M} €$ de royalties, un pactole que le directeur des sciences du vivant décide de consacrer à l'installation de l'Institut Neurospin ${ }^{25}$. En

22 Entretien de Jacques Grassi réalisé par 0. Frossard, le 2 déc. 2014 (archives CEA-FAR)

${ }^{23}$ Entretien de Michel Suscillon avec Odile Frossard, 19 nov. 2014 (archives CEA-FAR).

${ }^{24}$ André Syrota, (archives CEA-FAR).

${ }^{25}$ Entretien de Jacques Grassi réalisé par Odile Frossard, le 2 déc. 2014 (archives CEA-FAR). effet, outre la biologie structurale, l'autre axe de développement privilégié par la Direction des sciences du vivant concerne l'imagerie fonctionnelle. Inauguré en 2006 au CEN-Saclay dirigé par Denis Le Bihan, l'Institut d'imagerie biomédicale (Neurospin) est dédié à l'étude du cerveau. Ce plateau technique inter-organismes et interdisciplinaire, quasi unique au monde, permet de réunir physiciens, informaticiens, biologistes, cliniciens, neurologues et psychiatres comportementalistes autour des plus récentes techniques d'imagerie fonctionnelle, comme la magnétoencéphalographie (MEG) apte à mesurer les champs magnétiques les plus faibles induits par l'activité neuronale. Neurospin permet à des chercheurs comme Stanislas Dehaene et Jean-Pierre Changeux de décrire l'embrasement cortical, source de l'information consciente mémorisée et traitée par l'Homo sapiens, ou aux cliniciens de l'Institut du cerveau et de la moelle épinière (ICM) de la Salpêtrière de comprendre le fonctionnement du cerveau normal et de traiter ses pathologies [19].

C'est ainsi qu'au début du vingt et unième siècle, la Direction des sciences du vivant de ce qui est devenu le Commissariat à l'énergie atomique et aux énergies renouvelables (DSV-CEA) devient un modèle pour la réorganisation la recherche. À l'Éducation nationale, soucieux de moderniser ses modes de fonctionnement dans un espace mondialisé, le directeur de la recherche et de la technologie, Bernard Bigot ${ }^{26}$, confie à Gilles Bloch qui avait travaillé aux côtés d'André Syrota au SHFJ, le soin de mettre en place l'Agence nationale de la recherche (ANR) chargée de superviser l'ensemble de la recherche publique en France ${ }^{27}$. En 2008, pour sa part, André Syrota se voit proposer la direction de l'Inserm devenue vacante à la suite du départ de Christian Bréchot. Dans la lettre de mission qu'il soumet à l'aval de la ministre chargée de la recherche, Valérie Pécresse, il préconise l'installation d'une Alliance pour les sciences du vivant (AVIESAN, Alliance pour les sciences de la vie et de la santé) destinée à coordonner l'activité des grands établissements scientifiques œuvrant dans le domaine des sciences de la vie y compris au niveau international ${ }^{28}$. Assisté de Thierry Damerval qui fut son adjoint à la DSV-CEA ${ }^{29}$, il installe en 2009 à l'Inserm une série d'Instituts thématiques multiorganismes (les ITMO) inspirés du modus operandi adopté

\footnotetext{
${ }^{26}$ Premier directeur de l'ENS-Lyon, Bernard Bigot est nommé Haut Commissaire à l'énergie atomique en 2003.

${ }^{27}$ Après son passage au ministère de la Recherche, Gilles Bloch a assuré la direction de la DSV-CEA jusqu'en 2015. II préside aujourd'hui la communauté universitaire Paris-Saclay.

${ }^{28}$ Note à Valérie Pécresse, ministre de la Recherche, 2008 (archives Syrota, http:// www.histrecmed.fr/images/Inserm-AS.pdf)

${ }^{29}$ Entretiens de T. Damerval avec l'auteur réalisés en février et avril 2015.

(voir : http://www.histrecmed.fr/index.php?option=com_content\&view=article\&id=
} 288:thierry-damerval\&catid=8:entretiens). 
au Commissariat quelques années auparavant ${ }^{30}$. Ainsi, Jacques Grassi l'homme du test prions se retrouve chargé de l'ITMO Biologie-santé technologies pour la santé (ITS) responsable du lancement de plusieurs start-ups, exemple probablement le plus abouti d'un nouveau mode de management de la recherche dans notre pays ${ }^{31}$. $\diamond$

Physics of the radiations and life sciences - The Commissariat à l'énergie atomique (CEA) and the biomedical research, a historic overview

\section{LIENS D'INTÉRÊT}

Les auteurs déclarent n'avoir aucun lien d'intérêt concernant les données publiées dans cet article.

\section{RÉFÉRENCES}

1. Kohler RE. Partners in science. Foundations and naturel scientists. Chicago : Chicago University Press, 1991.

2. Morange M. Histoire de la biologie moléculaire. Paris : La Découverte, 1991.

3. Pinell P. Naissance d'un fléau. Histoire de la lutte contre le cancer en France 1890-1940. Paris : Métaillé, 1992.

4. Pinault M. Frédéric Joliot-Curie. Paris : Odile Jacob, 2000.

5. Heilbron J, Seidel R. Lawrence and his laboratory: a history of the Lawrence Berkeley laboratory. Oakland: University of California Press, 1989.

${ }^{30}$ Réorganisation de l'Inserm et coordination de la recherche biomédicale française. Mise en place des instituts thématiques, 2008 (archives Syrota, http://www.histrecmed.fr/images/ITMOS.pdf).

31 https://its.aviesan.fr/
6. Joliot F. Les applications biologiques des radioéléments. Bull ANM 1943 ; séance du 23 novembre 1943.

7. Chamak B. Cent ans de recherches en cancérologie : le rôle d'Antoine Lacassagne (1884-1971). Paris : Éditions Glyphe, 2011.

8. Picard JF. La fondation Rockefeller et la recherche médicale. Paris : PUF, 1999 : 206-9.

9. Gaudillières JP. Normal pathways: controlling isotopes and building biomedical research in postwar France. J Hist Biol 2006 ; 39 : 737-64.

10. Creager ANH, Santesmases MI. Radiobiology in the atomic age: changing research practices and policies in comparative perspective. J Hist Biol 2006 ; $39: 637-47$.

11. Picard JP, Mouchet S. La métamorphose de la médecine. Paris : PUF, 2009 : 153-7.

12. Tubiana M. Hommage au professeur Henri Jammet. Radioprotection 1996 ; $31: 459-67$.

13. Corvol P. François Morel (1923-2007), Hommage prononcé par le Professeur P. Corvol au Collège de France. La Lettre du Collège de France 2009 ; 25 : 43-44.

14. Fromageot $P$. Un volet de l'histoire des molécules marquées au CEA, la contribution de la biologie. Regard sur la biochimie $1994 ; 3$.

15. Veyret S. Tapis rouge pour un biologiste. Les Défis du CEA $1997 ; 63$.

16. Creager ANH. Life atomic: a history of radioisotopes in science and medicine. Chicago : University of Chicago Press, 2013.

17. Kellershohn C. Positron-emitting radionucleids in the study of metabolic and physiopathologic mechanisms. BrJ Radiol 1981; 54 : 91-102.

18. Yon-Kahn J. Histoire de la science des protéines. Les Ulis : EDP Sciences, 2006.

19. Griset P, Picard JF. L'atome et le vivant. Histoire d'une recherche issue du nucléaire. Paris: Cherche Midi, 2015 : 122 et sq.

\section{TIRÉS À PART}

J.F. Picard

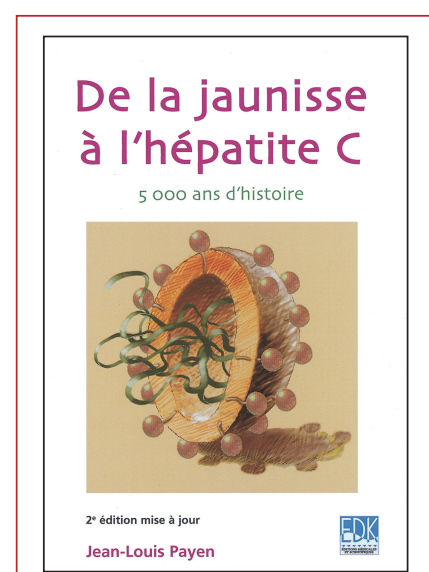

ISBN : 978-2-8425-4136-1 128 pages a jaunisse est un symptôme facilement identifiable ; il paraissait bien naturel que l'homme, confronté à une modification de la couleur de ses yeux et de sa peau ait de tous temps recherché les causes de cette transformation.

II n'est donc pas surprenant que le premier traité de médecine, écrit 3000 ans avant J.C. par un médecin sumérien, décrive déjà la jaunisse. À chaque époque de l'histoire de la médecine, les praticiens, influencés par les concepts médicaux de leur temps, attribuèrent une ou plusieurs explications particulières à ce symptôme. Ainsi, du démon Ahhâzu des Sumériens à la sophistication des biotechnologies qui permirent la découverte du virus de l'hépatite $C$, le lecteur cheminera sur une période de 5000 ans au travers des différents continents.

Ici encore, l'histoire se révèle une formidable source de réflexion : le foie souvent impliqué dans l'apparition des jaunisses est-il le siège de l'âme?

Les expérimentations humaines chez des volontaires ou chez des enfants handicapés mentaux étaient-elles justifiées pour permettre la découverte des virus des hépatites?

Le formidable développement de la transfusion sanguine, des vaccinations, mais aussi de la toxicomanie explique-t-il les épidémies d'hépatites du XXe siècle?

Autant de questions qui sont abordées dans ce livre passionnant et accessible à tous.

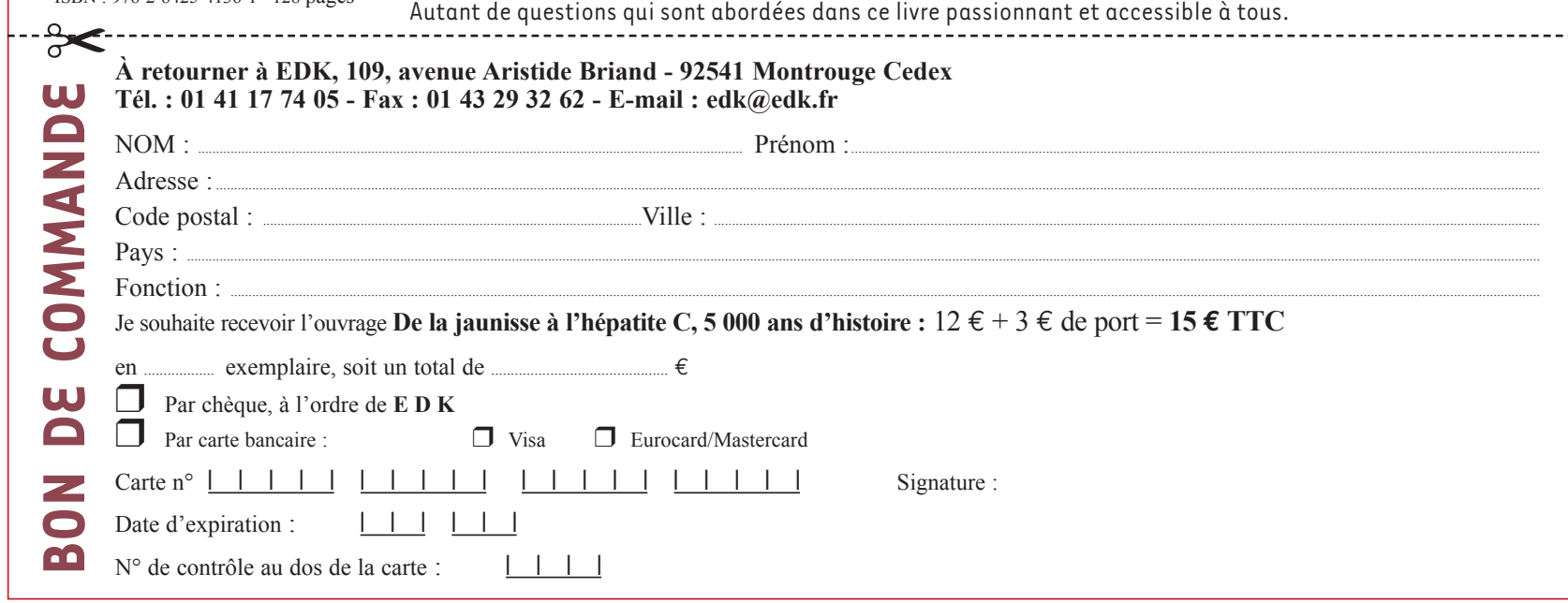

